University of South Carolina

Scholar Commons

$12-6-1993$

\title{
Neutron reflection interferometry: physical principles of surface analysis with phase information
}

Vladimir Gudkov

University of South Carolina - Columbia, gudkov@sc.edu

G. I. Opat

A. G. Klein

Follow this and additional works at: https://scholarcommons.sc.edu/phys_facpub

Part of the Physics Commons

Publication Info

Published in Journal of Physics: Condensed Matter, Volume 5, Issue 49, 1993, pages 9013-9024.

Gudkov, V.P., Opat, G.I., and Klein, A.G. (1993). Neutron reflection interferometry: physical principles of surface analysis with phase information. Journal of Physics: Condensed Matter, 5(49), 9013-9024. doi: 10.1088/0953-8984/5/49/004

(C) 1993 IOP Publishing Ltd.

This Article is brought to you by the Physics and Astronomy, Department of at Scholar Commons. It has been accepted for inclusion in Faculty Publications by an authorized administrator of Scholar Commons. For more information, please contact digres@mailbox.sc.edu. 


\title{
Neutron reflection interferometry: physical principles of surface analysis with phase information
}

\author{
V P Gudkov, G I Opat and A G Klein \\ School of Physics, University of Melboume, Parkville, Victoria 3052, Australia
}

Received 14 June 1993, in final form 23 September 1993

\begin{abstract}
It is shown that the analysis of surface layers by neutron reflection interferometry is considerably enhanced by performing the reflectometry with phase information. We discuss two methods of providing such information. One method involves physically adding an extra reference layer, whose amplitude and phase are known theoretically. The other uses the Lloyd's mirror configuration, in which a directly propagating ray that interferes with the reflected ray supplies the phase information. The methods have much in common with holography.
\end{abstract}

\section{Introduction}

There are many different ways to study the structure of matter using particle scattering. Here we discuss neutron surface reflection, which is capable of determining the surface matter distribution [1] (for recent discussions of this method see [2-7]). The main idea of this method relates to coherent low-energy neutron propagation through matter. It is well known that the propagation of a neutron beam can be described by geometrical optics using the refractive index

$$
n=1+\frac{\lambda^{2}}{2 \pi} N f
$$

where $\lambda$ is the neutron wavelength, $f$ is the forward scattering amplitude, and the $N$ is the nuclear number density [8]. Note that, in this paper, we do not consider ferromagnetic surfaces and, as a consequence, spin is ignored.

As we consider only low-energy neutrons, the forward scattering amplitude for most nuclei is constant and can be described in terms of the scattering length $b(b=-f(0))$. At these energies, non-resonant s-wave scattering is usually dominant, the scattering by other partial waves making corrections no greater than 1 in $10^{3}$. From (1) for the case $n<1$, neutrons are totally externally reflected by the surface, provided $\cos \Theta \geqslant n$, (where $\Theta$ is the glancing angle). Therefore, one can consider three different regimes of neutron reflection: total neutron reflection, ordinary neutron reflection and weak neutron reflection. Total reflection is interesting because it is very sensitive to the surface structure, has the highest intensity of the reflected beam and can be described analytically using a semiclassical approximation (see section 3). Weak reflection (or reflection with a large glancing angle) can be described by a form of perturbation theory applicable to continuous spectra (see section 4) and can be useful for understanding qualitatively ordinary reflection, which is a transition region between these two extreme cases.

In the analysis of neutron reflection data, numerical fitting of density profile models to the data is usually employed (see, for example, $[9,10]$ and references therein). However, this 
method does not suffice to determine a totally unknown surface structure by purely numerical methods because the phase is unknown. Furthermore, it is impossible to distinguish between real surface density fluctuations and numerical approximation noise. There are proposals $[11,12]$ to determine neutron reflection phases using additional measurements of neutron absorption rates. However, for this procedure to work well the energy dependence of the reflection and transmission coefficients must be known to high accuracy over a broad energy range. In this paper we discuss the possibility of interference measurements which allow the experimental determination of the phases and consequently permit a complete inversion of the problem, leading to a unique determination of the density profile.

For completeness, let us briefly review two widely used numerical methods. The first is related to the numerical solution of the Schrödinger equation for some model potential (the density distribution). The density distribution is obtained as a result of a fit to the experimental reflectivity using the free parameters of the model density distribution. The second method is a multilayer approximation (see, for example, the a description of the method [13] and a recent review of its application to neutron refiection [9]) for the determination of the surface structure. The reflection from each layer is parametrized by a characteristic matrix which depends on the boundary conditions for the layer. The resultant reflectivity for many layers is then given as a product of the characteristic matrices. The model of the surface which is used in conjunction with the multilayer approach contains free parameters. These parameters are determined by fitting the predictions of the model to the experimental data.

As mentioned above, all numerical approaches need an a priori model for the density distribution. In this paper we are interested in model-independent methods of obtaining the density distribution from the experimental data.

Before discussing the relationship between the surface structure and the reflection amplitude $R$ (where $|R|^{2}$ is the ratio of incident to reflected beam intensities), let us recall some general aspects of the scattering process. As is well known, the differential cross section for low-energy scattering is given by

$$
\frac{\mathrm{d} \sigma}{\mathrm{d} \Omega}=|b|^{2}|F(a)|^{2}
$$

where the form factor, $F(q) \sim \int \exp (\mathrm{i} q \cdot r) N(\boldsymbol{r}) \mathrm{d} r$. Here $N(\boldsymbol{r})$ is the target number density, $q$ is the neutron momentum transfer

$$
|\boldsymbol{q}|=2 k \sin \Theta
$$

and $k=2 \pi / \lambda$ is the neutron wavevector. Since $F(q)$ is simply the Fourier transform of the density, $N$, the direct procedure of obtaining the $N(r)$ distribution from the experimental cross section is by Fourier inversion.

The process of neutron reflection is more complicated because it is effectively a multiscattering coherent process. Therefore the integral relation between the form factor and the density is more complicated and, in particular, is dependent on the density distribution. To show this, we give the expression for the corresponding form factor in the WKB approximation:

$$
F \sim \int_{0}^{-\infty} \exp \left(\mathrm{i} k y \sqrt{\sin ^{2} \Theta-\frac{4 \pi b}{k^{2}} N(y)}\right) N(y) \mathrm{d} y .
$$

Here $y$ is a coordinate normal to the surface. In general, it is impossible to find an exact transformation to invert the reflection coefficient into the density distribution. 
The purpose of this paper is to find some special cases for which there is a possibility of finding a model-independent density distribution in the vicinity of the surface using the experimental reflection coefficient $|R|^{2}$. We will show that there are two extreme cases where one can obtain analytical solutions for the density distributions: the case of total reflection and the case of weak reflection.

\section{Spatial resolution}

Firstly, let us consider some simple examples (which have analytical solutions) to estimate the characteristic spatial resolution for neutron reflection. We are interested in total reflection or in reflection in the vicinity of total reflection (in terms of the parameters), where the neutron intensity is maximal. For an isotropic sample at small glancing angles $(\Theta \ll 1)$ the relationship between the angle of total reflection $\Theta_{\mathrm{cr}}$, and minimal wave length $\lambda_{\mathrm{cr}}$ is

$$
\Theta_{\mathrm{cr}}=(N b / \pi)^{1 / 2} \lambda_{\mathrm{cr}}
$$

or in terms of the momentum transfer

$$
q_{\mathrm{cr}}=4(\pi N b)^{1 / 2}
$$

The parameters $\Theta_{\mathrm{cr}}, \lambda_{\mathrm{cr}}$ and $q_{\mathrm{cr}}$ are called critical parameters, and the region in the vicinity of these parameters is the critical region.

To understand the resolution achievable in neutron refiection, let us compare the reflection coefficient from a surface with a rectangular density step with that for a smooth behaviour at the surface. For the first case

$$
\begin{array}{ll}
N(y)=0 & y>0 \\
N(y)=N_{0} & y \leqslant 0
\end{array}
$$

and for the second case

$$
N(y)=\frac{1}{2} N_{0}[1+\tanh (\pi y / \Delta)]
$$

where $\Delta$ is a diffuseness parameter $\left(N(-\Delta)=0.002 N_{0}\right.$ and $\left.N(\Delta)=0.998 N_{0}\right)$. We use a Fermi potential to describe neutron propagation in matter:

$$
V(y)=\frac{2 \pi \hbar^{2}}{m} b N(y)
$$

This potential describes well the multiple coherent scattering propagation of low-energy neutrons in media (for a detailed discussion see, for example, [8]).

To find the reflection coefficient we solve the one-dimensional Schrödinger equation for the corresponding potential. We find $|R|^{2}=1$ for both density distributions, provided $q<q_{\mathrm{cr}}$ (total reflection). For $q \geqslant q_{\mathrm{cr}}$ the rectangular step distribution leads to

$$
\left|R_{\mathrm{r}}\right|^{2}=\frac{2-\left(q_{\mathrm{cr}} / q\right)^{2}-2 \sqrt{1-\left(q_{\mathrm{cr}} / q\right)^{2}}}{2-\left(q_{\mathrm{cr}} / q\right)^{2}+2 \sqrt{1-\left(q_{\mathrm{cr}} / q\right)^{2}}}
$$


whereas the smooth distribution leads to

$$
\left|R_{\mathrm{s}}\right|^{2}=\left(\frac{\sinh \left[\left(1-\sqrt{1-\left(q_{\mathrm{cr}} / q\right)^{2}}\right)(q \Delta / 4)\right]}{\sinh \left[\left(1+\sqrt{1-\left(q_{\mathrm{cr}} / q\right)^{2}}\right)(q \Delta / 4)\right]}\right)^{2} .
$$

From the last two equations we conclude that $\left.\left|R_{\mathrm{r}}\right|^{2} \rightarrow{ }_{\mid} R_{\mathrm{s}}\right|^{2}$ in the vicinity of a critical point when

$$
\Delta \ll \Delta_{c h} \equiv 4 / q_{c r} \equiv(\pi b N)^{-1 / 2}
$$

where $q=q_{\mathrm{cr}}$ for the total reflection case. Equation (12) defines a characteristic surface diffuseness, $\Delta_{\mathrm{ch}}$, which sets the scale of spatial resolution. For typical values, $b \sim 10^{-12} \mathrm{~cm}$ and $N \sim 10^{22} \mathrm{~cm}^{-3}$, we find $\Delta_{\mathrm{ch}} \sim 500 \AA$, so that in the vicinity of total reflection it is possible to study surfaces with a resolution of about $500 \AA$.

Moreover, the different density distributions lead to different energy (or wavelength) dependence of the reflection coefficients. In the vicinity of the critical point, the ratio of derivatives is

$$
\eta=\frac{\partial\left|R_{\mathrm{r}}\right|^{2} / \partial \lambda}{\partial\left|R_{\mathrm{s}}\right|^{2} / \partial \lambda}=\left(\frac{\Delta}{\Delta_{\mathrm{ch}}}\right) \operatorname{coth}\left(\frac{\Delta}{\Delta_{\mathrm{ch}}}\right) .
$$

Figure 1 shows the reflection coefficients for abrupt and smooth surfaces (equations (10) and (11)). We see that a sharp break in the slope of an experimental reflection coefficient determines $q_{\mathrm{cr}}$. The slope of the reflection coefficient data for $q \leqslant q_{\mathrm{cr}}$, together with (13), yields the surface diffuseness parameter $\Delta$.

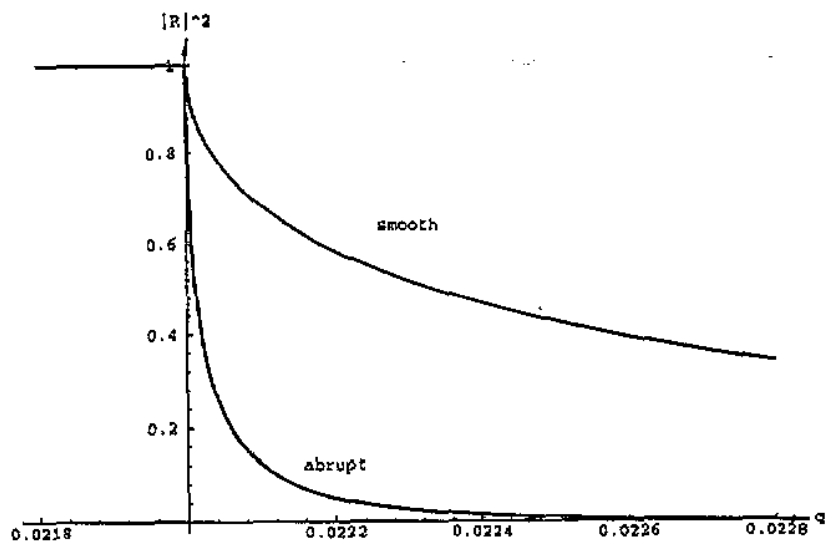

Figure 1. Reflection coefficients for abrupt and smooth $(\Delta=1000 \AA)$ surfaces.

It should be noted that to investigate a density distribution with a resolution better than $\Delta_{\text {ch }}$, one should use weak reflection because the spatial resolution is proportional to the reciprocal of the neutron momentum transfer (see (12)).

\section{The density distribution near the surface: total reflection}

We often need to determine the structure of a surface in which an 'impurity' is diffusely embedded in an otherwise well understood abrupt surface. The full solution of this reflection 
problem is difficult; however, if the main part of the surface is regarded as known, the determination of the structure of the 'impurity' layer is tractable. We now address this problem.

Consider a compound sample consisting of two different substances: one has $N_{0}(y)$, the density distribution with $\Delta \ll \Delta_{0}\left(\Delta_{0}=\left(\pi b_{0} N_{0}(-\infty)\right)^{-1 / 2}\right.$, where $b_{0}$ is the scattering length for this substance); the other has $N(y)$, the density distribution with $\Delta>\Delta_{0}$. We are interested in the case

$$
b_{0} N_{0}(y) \gg b N(y)
$$

where $b$ is the scattering length of the second substance. The first substance is considered to be the main one, the second to be a small admixture. Due to the small diffuseness of the main density distribution one can describe it using the rectangular step distribution. The purpose of this section is to obtain an analytic dependence of the reflection coefficient on the small density distribution, $N(y)$. To do this we use the WKB approximation. There are three different regions of the parameters: (i) $q<q_{0}$, where $q_{0}$ is critical neutron transfer momentum for only the $N_{0}(y)$ density; (ii) $q>q_{\Sigma}$, where $q_{\Sigma}$ is the critical neutron transfer momentum for the total density $N_{0}(y)+N(y)$; and (iii) $q_{0}<q<q_{\Sigma}$.

For the first region we have total reflection. For the second we have the usual reflection. We will not discuss these two regions further, since for them the reflection coefficient $R$ is dependent only on the average value, $N(y)$, and its derivative $\partial N(y) /\left.\partial y\right|_{y=0}$. The last region is the most interesting, as the total reflection in this region, $|R|^{2}=1$, and therefore $R=\exp (\mathrm{i} \delta$ ). We calculate the phase shift $\delta$ using the wKB approximation for two different $N(y)$ profiles: (i) $N(y)$ increases in the vicinity of the surface and becomes a constant at a large distance from the surface; and (ii) $N(y)$ is large near the surface and becomes zero at large distances. From the point of view of the WKB approximation case (ii) needs a special approach near the point where $N(y)$ is maximal, since there are two close turning points. This special approach leads to long formulae, unless we exclude from consideration the vicinity of the maximal value of $N(y)$.

The standard WKB method gives

$$
R=\frac{1-\mathrm{i} \sqrt{1-\left(q_{0} / q\right)^{2}} \cot \left(\alpha+\frac{1}{4} \pi\right)}{1+\mathrm{i} \sqrt{1-\left(q_{0} / q\right)^{2}} \cot \left(\alpha+\frac{1}{4} \pi\right)}
$$

with

$$
\tan \frac{1}{2} \delta=-\sqrt{1-\left(q_{0} / q\right)^{2}} \cot \left(\alpha+\frac{1}{4} \pi\right)
$$

Here $q_{0}$ is the critical neutron transfer momentum for the density $N_{0}(y)$

$$
\alpha=\frac{1}{2} \int_{0}^{\xi} \sqrt{q^{2}-q_{0}^{2}-16 \pi b N(y)} d y
$$

and $\xi$ is a root of the equation

$$
q^{2}-q_{0}^{2}-16 \pi b N(y)=0 .
$$

We conclude from (16) that the experimental value of the phase shift $\delta$ determines $\alpha(q)$. This parameter is simply related to $N(y)$ by (17), giving the possibility that $N(y)$ may be obtained in a model-independent way. Hence we have a practical method of improving the numerical analysis of experimental neutron reflectivity data.

We must realize that no amount of analysis will yield surface features with spatial resolution better than $\Delta_{\mathrm{ch}}$. Due to this physical limitation all functions used in fitting the density distribution should be smooth on a scale $\sim \Delta_{\text {ch }}$. For example, fitting the numerical data of [10] should give a reasonably good description of the density distribution with a $\Delta_{\mathrm{ch}}$ resolution. 


\section{The density distribution near the surface: weak refiection}

Weak reflection (large glancing angle $\Theta$ ) may also be studied using the WKB approximation. However, when the neutron energy is much larger than the Fermi potential of the medium, a smooth density distribution near the surface leads to a real reflection coefficient, as in the case of a rectangular step density distribution (see (7)). This obvious result means that for large neutron energy a small variation in the density distribution leads to a small perturbation of the reflection coefficient, giving us an opportunity to apply perturbation theory to the weak reflection case.

It should be noted that the general inverse scattering problem for weak reflection (in particular for the reflection of electromagnetic waves) has been given much attention (see, for example, [14] and tive extensive references therein). According to the above paragraph, we shall discuss only the special case of neutron refiection in which the main contribution to the reffection amplitude may be treated as the well known reflection amplitude for a rectangular step density distribution. Therefore, we are interested in obtaining information about small unknown variations of the density distribution.

To calculate the weak reflection amplitude, $R$, let us consider the following density distribution (compare with (7)):

$$
\begin{array}{ll}
N(y)=0 & y>0 \\
N(y)=N_{0} & y \rightarrow-\infty .
\end{array}
$$

The boundary conditions for the wavefunction at $y=0$ yield a reflection amplitude

$$
R=\frac{\mathrm{i} q-2 \chi}{\mathrm{i} q+2 \chi}
$$

where $\chi=\psi^{\prime}(0) / \psi(0)$ and $\psi(y)$ is the solution of the Schrödinger equation (with the potential (9) for the density distribution (19)) for the $y<0$ region. Let us assume that we know the wavefunction $\psi_{0}(y)$ for the density distribution incorporated in the Hamiltonian $H_{0}$. A small variation of the density distribution yields an additional potential $V$. If $V \ll H_{0}$, one can solve the problem for the total Hamiltonian $H=H_{0}+V$ using continuous spectrum perturbation theory. If $\psi_{n}$ is the $n$th iterated wavefunction of the Lipman-Schwinger equation, the first iteration is

$$
\psi_{1}=\psi_{0}+\lim _{\epsilon \rightarrow 0} \frac{1}{E-H_{0}+\mathrm{i} \epsilon} V \psi_{0}
$$

where $E$ is the neutron energy. The logarithmic derivative of the wavefunction is now given by

$$
\begin{aligned}
x_{1}=\left[\psi_{0}^{\prime}(0)\right. & \left.+\int_{0}^{\infty} \mathrm{d} x\left(\left.\frac{\partial G_{0}(y, x)}{\partial y}\right|_{y=0}\right) V(x) \psi_{0}(x)\right] \\
& \times\left(\psi_{0}(0)+\int_{0}^{\infty} \mathrm{d} x G_{0}(0, x) V(x) \psi_{0}(x)\right)^{-1}
\end{aligned}
$$

where $G_{0}(y, x)$ is a Green function of the Hamiltonian $H_{0}$. 
The substitution of (22) into (20) gives the perturbation theory expression for the reflection amplitude:

$$
R_{1}=\frac{\mathrm{i} q-2 \chi_{1}}{\mathrm{i} q+2 \chi_{\mathrm{i}}} .
$$

At first sight the resulting expression for $R_{1}$ might seem complicated; however the procedure gives a simple analytic expression for the case of reflection by a rectangular step density distribution (equation (7)) together with an additional small distribution $N(y)$ of a species with scattering length $b$ (see the previous section). The analytic expressions for $\psi(y)$ and $G_{0}(y, x)$ for a rectangular step (using (22) and (23) to first order in density $\left.N(y)\right)$, yield

$$
R_{1}=R_{0}+\eta\left(1+\sqrt{1-\left(q_{0} / q\right)^{2}}\right) .
$$

Thus

$$
\left|R_{1}\right|^{2}=\left|R_{0}\right|^{2}+\left|R_{0}\right| \frac{1}{2}\left(1+\sqrt{1-\left(q_{0} / q\right)^{2}}\right)|\eta| \cos \delta_{\eta}
$$

where

$$
R_{0}=\frac{1-\sqrt{1-\left(q_{0} / q\right)^{2}}}{1+\sqrt{1-\left(q_{0} / q\right)^{2}}}
$$

Here $q_{0}$ is the critical neutron momentum transfer for the rectangular step potential. We define $k_{0}$ by

$$
k_{0} \equiv \sqrt{q^{2}-q_{0}^{2}} .
$$

Because

$$
\eta=|\eta| \exp \left(\mathrm{i} \delta_{\eta}\right)=\frac{4 \mathrm{i} \pi b}{k_{0}} \int_{-\infty}^{0} N(y) \exp \left(\mathrm{i} k_{0} y\right) \mathrm{d} y
$$

we find

$$
\operatorname{Re} \eta=|\eta| \cos \delta_{\eta}=-\frac{4 \pi b}{k_{0}} \int_{-\infty}^{0} N(y) \sin \left(k_{0} y\right) \mathrm{d} y .
$$

From ( $28 b)$ one concludes that in the case of weak reflection one may obtain the density distribution near a surface by using Fourier sine inversion for the experimental parameter $|\eta| \cos \delta_{\eta}$ of $(25)$.

Equations (24)-(28) agree with the result of the general discussion (equation (4)) in the introduction. Perturbation theory leads to the simple relation (28) between the experimental value $\eta$ and the unknown density distribution $N(y)$. However, it would be difficult to apply this relation to the practical extraction of the density distribution from the experimental data since the inversion (28) requires the measurement of $\eta$ (or $R_{1}$ ) over a wide range of momentum transfer. Nevertheless, weak reflection may be used as an additional method to verify the density distribution $N(y)$ obtained from total reflection data. 


\section{Using an artificial layer to obtain phase information}

We consider the measurement of the neutron reflection coefficient of a surface, with and without the addition of an artificially applied surface layer. This method is normally used in optics; however it has been also suggested that it could be used in refiection studies [15]. On the basis of the numerical solution of a surface model it was concluded in that paper that there are two advantages in using speckle holography: first, it may be possible to use the data to distinguish between several previously ambiguous solutions; second, it is possible to analyse the experimental data using Fourier transformations. The details of this method of analysis are given below.

The modification of the reflection by the additional surface layer, whose contribution to reflection is understood, can provide us with the needed reference phase.

This possibility is related to the results of section 2 . If there is a layer on the surface with a thickness $l \ll \Delta_{\text {ch }}=4 / q$ (see (12)), the layer may be described by the $\delta$-function potential

$$
V_{\mathrm{L}}=-\frac{2 \pi \hbar^{2}}{m} N f(0) l \delta(y)
$$

Here we use the general expression for the forward scattering amplitude $f(0)$ to allow for absorption as well. It should be noted that in accordance with the restriction $l \ll \Delta$ this approach is best suited to strong (or total) reflection.

Using (20) as the expression for the reflection coefficient with the surface layer absent, and then using the boundary conditions for the wavefunction in the case of a $\delta$-shape potential (29), we obtain the following expression for the reflection coefficient with the layer:

$$
R_{\mathrm{L}}=\frac{\mathrm{i} R+\beta(R+1)}{\mathrm{i}-\beta(R+1)}
$$

where

$$
\beta=-4 \pi N f(0) l / q .
$$

If we resolve $\beta$ into its real and imaginary parts $\xi$ and $\gamma$, i.e. $\beta=\xi+\mathrm{i} \gamma$, we find in terms of the complex $f(0)$ that $\xi=4 \pi N b l / q$ and $\gamma=-4 \pi N l(\operatorname{Im} f(0)) / q$. Then, from (31), one obtains

$\left|R_{\mathrm{L}}\right|^{2}=\frac{r^{2}+\left(\xi^{2}+\gamma^{2}\right)\left(r^{2}+1+2 r \cos \delta\right)-2 \gamma r^{2}-2 \xi r \sin \delta-2 \gamma r \cos \delta}{1+\left(\xi^{2}+\gamma^{2}\right)\left(r^{2}+1+2 r \cos \delta\right)-2 \xi r \sin \delta+2 \gamma r \cos \delta+2 \gamma}$

where $R=r \exp (\mathrm{i} \delta)$.

For neutron reflection without absorption this expression leads to

$$
\left|R_{\mathrm{L}}\right|^{2}=\frac{r^{2}+\xi^{2}\left(1+r^{2}+2 r \cos \delta\right)-2 \xi r \sin \delta}{1+\xi^{2}\left(1+r^{2}+2 r \cos \delta\right)-2 \xi r \sin \delta} .
$$

For the case $\xi \ll 1,(33)$ has the simple form

$$
\left|R_{\mathrm{L}}\right|^{2} \simeq|R|^{2}\left[1+2 \xi\left(r-\frac{1}{r}\right) \sin \delta\right]
$$


A strongly absorbing layer $(|\gamma| \gg|\xi|)$ leads to a modification of the previous total reflection (provided $|R|^{2}=r^{2}=1$ ), namely

$$
\left|R_{\mathrm{L}}\right|^{2}=\frac{1+2 \gamma(\gamma-1)(1+\cos \delta)}{1+2 \gamma(\gamma+1)(1+\cos \delta)} .
$$

This expression yields simple oscillations of the reflection coefficient, if $|\gamma| \ll 1$ :

$$
\left|R_{\mathrm{L}}\right|^{2} \simeq 1-4 \gamma(1+\cos \delta) \text {. }
$$

We see that the separate measurernents of the intensity of neutron beams reflected from the original surface and the artificially layered surface yields the phase of the reflection coefficient.

\section{Numerical examples}

Using the above expressions, one may predict experimental results for neutron reflection for different cases. For this purpose we will consider the case of neutron reflection with momentum transfer $q \sim(0.01-1.0), \AA^{-1}$ from a nickel surface $\left(N b=9.41 \times 10^{-6} \AA^{-2}\right)$ with a specified admixture.

For the total reflection with the admixture density distribution

$$
N(y)=N y^{2} / a^{2}
$$

the results of the WKB approximation in section 3 (see (16)) are shown in figures 2 and 3 for admixtures with $N b=5 \times 10^{-7} \AA^{-2}$ for the case $a=1000 \AA$ and $a=100 \AA$. One can see that the phase of reflection is very sensitive to the amount of the admixture and its spatial distribution.

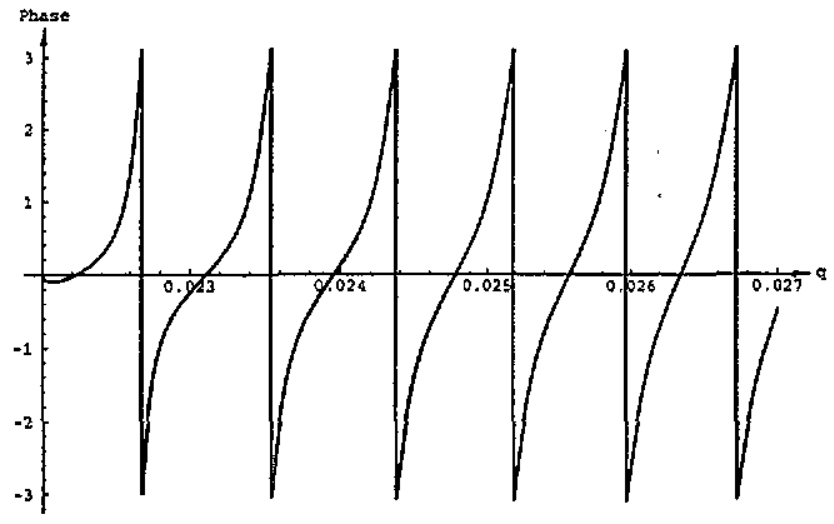

Figure 2. Phase of reflection. The admixture parameters are $\mathrm{Nb}=5 \times$ $10^{-7} \AA^{-2}$ and $a=1000 \AA$.

Weak reflection (section 4) leads to a modification of the reflection coefficient for the rectangular substrata distribution of nickel (equation (26) and figure 4) admixed with the density distribution

$$
N(y)=N \sin (-\pi y / a)
$$



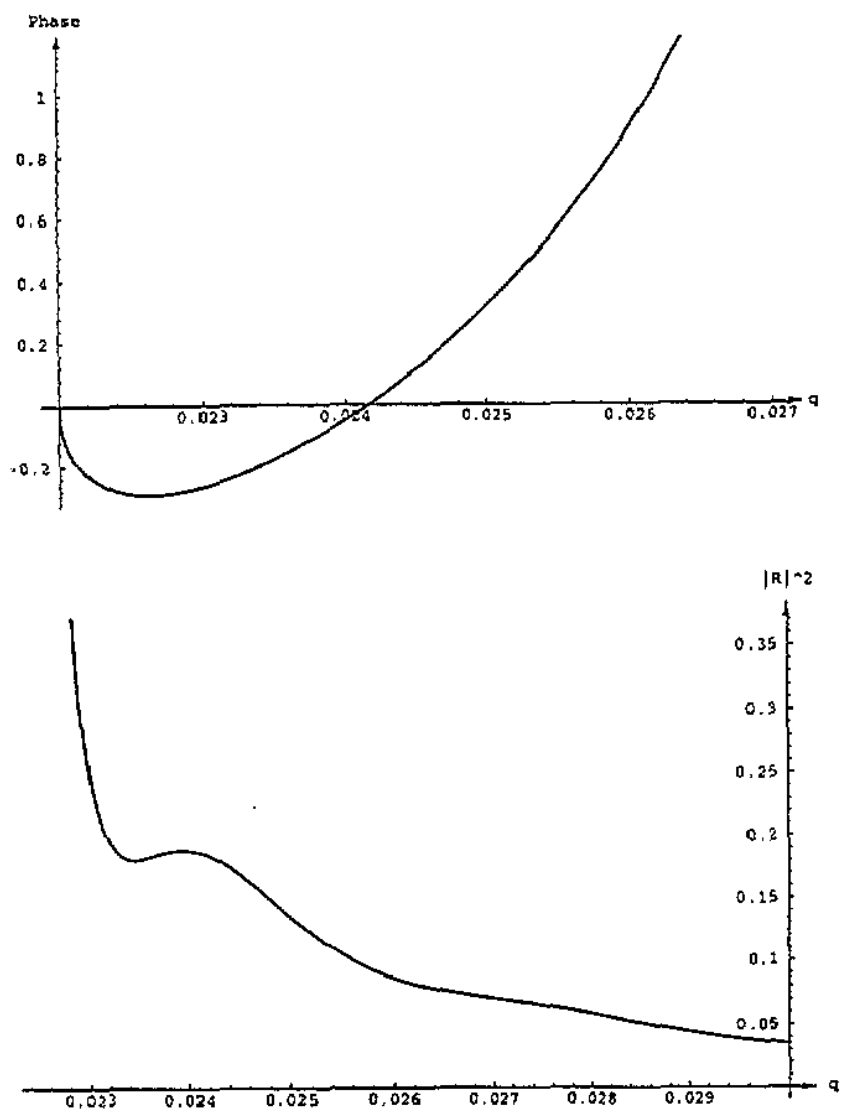

Figure 3. Phase of reflection. The admixture parameters are $\mathrm{Nb}=5 \times$ $10^{-7} \mathrm{~A}^{-2}$ and $a=100 \mathrm{~A}$.

Figure 4. Weak reflection $(\mathrm{Nb}=$ $10^{-6} \AA^{-2}$ and $\left.a=1000 \AA\right)$.

for $y \in[0,-a]$. The reflection coefficient (25) for the parameters $N b=10^{-6} \AA^{-2}$ and $a=1000 \AA$ is shown in figure 4. This example shows that weak reflection is also sensitive to the admixture distribution.

Finally, let us consider the case of layered surfaces. We accept (11) as a model for the non-layered nickel surface with the parameter $\Delta=1000 \AA$. This reflection coefficient is shown in figure 5. The modifications of this coefficient by a thin layer $(l=10 \AA$ ) with a pure imaginary neutron scattering amplitude are shown in figure 5 , i.e. for $b=0$ and $N \operatorname{Im} f(0)=9.4 \times 10^{-6} \AA^{-2}$. It is obvious that this modification may also be used to obtain information about the phase of reflection (see (32) and (35)).

\section{Phase information using the Lloyd mirror configuration}

The Lloyd mirror interferometer $[16,17]$ (see figure 6 ) can provide phase information for neutron reflection. The direct neutron beam can be considered as a reference beam for the reflected one. The total intensity of the sum of these two beams, $I_{\text {sum, }}$, provides the interference pattern on the screen:

$$
I_{\text {sum }}=1+|R|^{2}+2|R| \cos \left(\delta+\delta_{\text {geom }}\right) .
$$



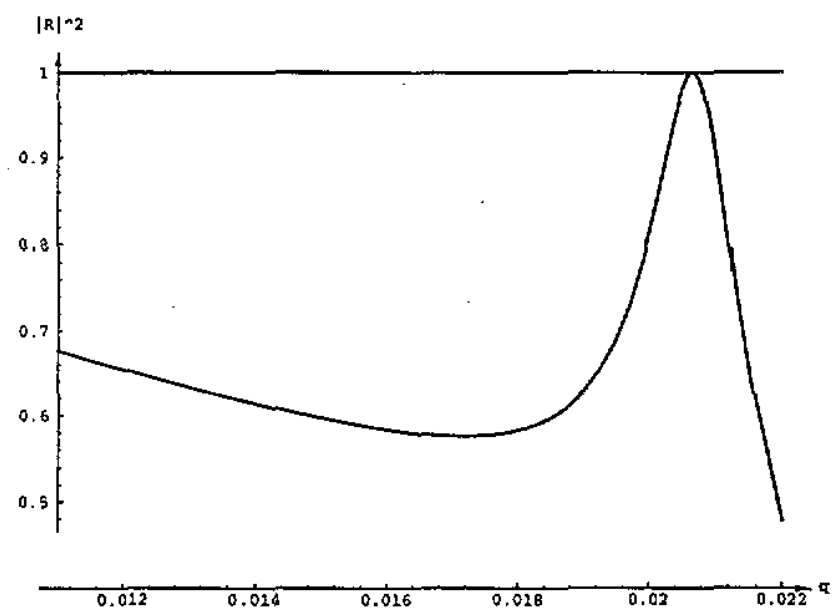

Figure 5. The horizontal line, $|R|^{2}=1$, shown the reflection coefficient for no layer, i.e. total reflection. The curve, which defers significantly from this total reflection line, shows the effect of a thin absorptive layer, for which the thickness, $l=10 \AA$, scattering length $b=0$, and $N \operatorname{Im} f(0)=9.41 \times 10^{-6} \AA^{-2}$.

Here we assume that the intensity of the reference beam is one (i.e. the direct and incident beams have equal amplitude) and use the standard definition for the reflection amplitude $R=|R| \exp \delta$. The geometric phase shift due to the difference in the paths of the reference and reflected beams has the simple expression (for notation see figure 6)

$$
\delta_{\text {geom }}=k\left(\sqrt{L^{2}+(x+d)^{2}}-\sqrt{L^{2}+(x-d)^{2}}\right)
$$

and for the case $x, d \ll L$ is proportional to the angle $\alpha$ :

$$
\delta_{\text {geom }}=2 k d \alpha \text {. }
$$

Figure 7 shows the interference pattern for neutron reflection with $\lambda=2 \AA$ on the surface with a density distribution according to (11) with parameters $q_{0}=0.022$ and $\Delta=1000 \AA$ on an interferometer with $d=1 \mathrm{~cm}$ and $L=5 \mathrm{~m}$.

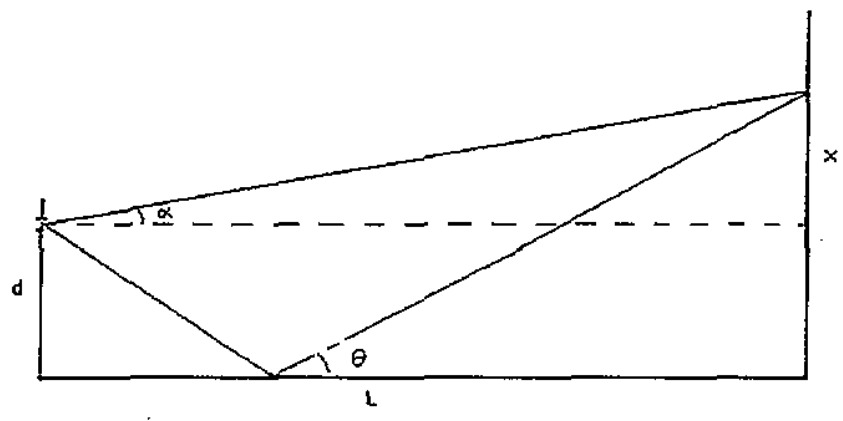

Figure 6. The Lloyd mirror interferometer for neutron reflection showing the direct and reflected rays. The height of the slit above the reference surface is $d, L$ is the slit-to-detector distance, and $x$ is the detector height in the detector plane, measured from the plane of the mirror.

\section{Conclusion}

In this paper we have considered the reflection of neutrons by surfaces in several ways, each of which shows an effect of the phase of reflection. In turn this enhances our ability to determine of the surface structure. 


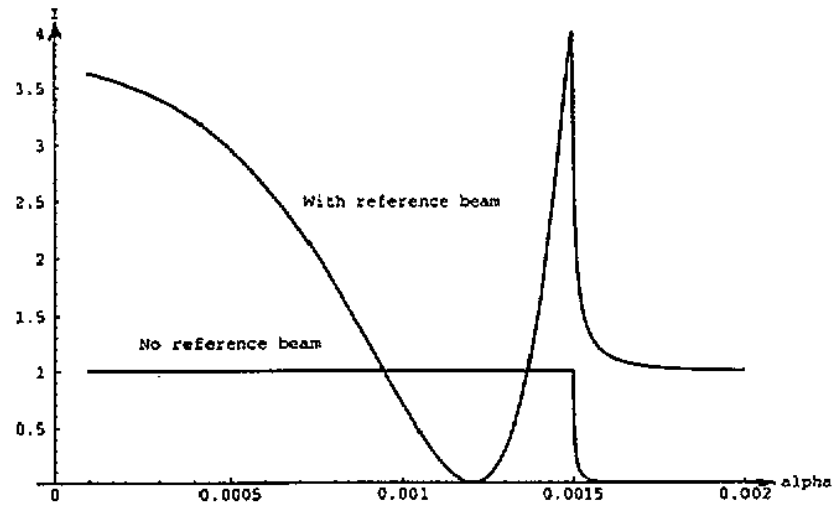

Figure 7. The intensity pattem for the Lloyd mirror interferometer. For reference, the intensity is also shown when the reference beam is absent.

The first method considers the contribution to reflection by the deviation of the structure of the surface from a fully understood simple surface structure. The limits to the resolution of this method were also found.

A second method shows how the artificial addition of a surface layer of known properties modifies the reflection of the unknown underlying surface, thereby providing extra phase information.

A third method makes use of a direct reference ray (in a Lloyd mirror configuration) to provide, in a holographic-like way, the reference phase.

\section{Acknowledgments}

The authors are indebted to the Australian Research Committee (ARC) for support.

\section{References}

[1] Penfold J and Thomas R K 1990 J. Phys.: Condens. Matter 21369

[2] Mâaza M, Pardo B and Bridou F 1993 J. Appl. Crystallogr. 26327

[3] Mâaza M, Sella C, Ambroise J P, Kâabouchi M, Milôche M, Wehling F and Cros M 1993 J. Appl. Crystallogr. 26334

[4] Mâaza M, Ziang Z, Samuel F, Famoux B and Vidal B 1992 J. Appl. Crystallogr. 25787

[5] Mâaza M and Pardo B 1993 J. Opt. Commun. submitted

[6] Måaza M 1990 Enrico Fermi Int. School of Physics: Industrial and Technological Applications of Neutrons, 19-29 June 1990. Villa Marigolla, ltaly

[7] Mâaza M, Sella C and Kâabouchi M 1991 Ist Int. Symp. on Atomically Controlled Surfaces and Interfaces, November 19-22 1991. Tokyo. Japan

(8) Gurevich I I and Tarasov L V 1968 Low-Energy Neutron Physics (Amsterdam: North-Holland)

[9] Penfold J 1990 Inst. Phys. Conf. Series 107 (Bristol: Institute of Physics Publishing) p 213

[10] Sivia D S, Hamilton W A and Smith G S 1990 inst. Phys. Conf. Series 107 (Bristol: Institute of Physics Publishing) p 223

[11] Fiedeldey H, Lipperheide R, Leeb H and Sofianos S A 1992 Phys. Lett. 170A 347

[12] Lipperheide R et al 1993 Physica B at press

[13] Born M and Wolf E 1959 Principles of Optics: Electromagnetic Theory of Propagation, Interference and Diffraction of Light (Oxford: Pergamon)

[14] Lekner J 1987 Theory of Reflection (Dordrecht: Nijhoff)

[15] Sivia D S, Hamilton W A and Smith G S 1991 Physica B 173121

[16] Allman B E, Klein A G, Nugent K A and Opat G I 1993 Eur, $J$, Phys. at press

[17] Allman B E, Klein A G, Nugent K A and Opat G I 1993 Appl. Opt. at press 